\title{
Sociodemographic Characteristics Predicting Digital Health Intervention Use After Acute Myocardial Infarction
}

\author{
Lochan M. Shah ${ }^{1,2} \cdot$ Jie Ding ${ }^{1,2} \cdot$ Erin M. Spaulding ${ }^{2,3,4} \cdot$ William E. Yang ${ }^{1,2} \cdot$ Matthias A. Lee $^{5} \cdot$ Ryan Demo $^{5}$ • \\ Francoise A. Marvel ${ }^{1,3}$. Seth S. Martin ${ }^{1,2,4,5}$ (D)
}

Received: 5 October 2020 / Accepted: 4 January 2021 / Published online: 17 May 2021

(C) The Author(s), under exclusive licence to Springer Science+Business Media, LLC part of Springer Nature 2021

\begin{abstract}
Increasing evidence suggests that digital health interventions (DHIs) are an effective tool to reduce hospital readmissions by improving adherence to guideline-directed therapy. We investigated whether sociodemographic characteristics influence use of a DHI targeting 30-day readmission reduction after acute myocardial infarction (AMI). Covariates included age, sex, race, native versus loaner iPhone, access to a Bluetooth-enabled blood pressure monitor, and disease severity as marked by treatment with CABG. Age, sex, and race were not significantly associated with DHI use before or after covariate adjustment (fully adjusted OR 0.98 (95\%CI: 0.95-1.01), 0.6 (95\%CI: 0.29-1.25), and 1.22 (95\% CI: 0.60-2.48), respectively). Being married was associated with high DHI use (OR 2.12; 95\% CI 1.02-4.39). Our findings suggest that DHIs may have a role in achieving equity in cardiovascular health given similar use by age, sex, and race. The presence of a spouse, perhaps a proxy for enhanced caregiver support, may encourage DHI use.
\end{abstract}

Keywords Sociodemographic factors $\cdot$ Digital health $\cdot$ Hospital readmission $\cdot$ Myocardial infarction $\cdot$ mHealth $\cdot$ Health disparities

\author{
Abbreviations \\ DHI Digital health intervention \\ AMI Acute myocardial infarction \\ CABG Coronary artery bypass grafting \\ STEMI ST-elevation myocardial infarction
}

Associate Editor Navin Kumar Kapur oversaw the review of this article

Seth S. Martin

smart100@jhmi.edu

1 Johns Hopkins University School of Medicine, Baltimore, MD, USA

2 Digital Health Innovation Laboratory, Ciccarone Center for the Prevention of Cardiovascular Disease, Division of Cardiology, Department of Medicine, Johns Hopkins University School of Medicine, Baltimore, MD, USA

3 Johns Hopkins University School of Nursing, Baltimore, MD, USA

4 Johns Hopkins Center for Mobile Technologies to Achieve Equity in Cardiovascular Health (mTECH), an AHA SFRN Center for Health Technology and Innovation, Baltimore, MD, USA

5 Johns Hopkins University Whiting School of Engineering, Baltimore, MD, USA

\section{Introduction}

Readmission after AMI is common, and in many cases, potentially preventable through implementation of best practices $[1,2]$. These include comprehensive education, pre-discharge follow-up planning, and personalized reinforcement of medication adherence [3]. Readmission reduction has consequently become a priority for several countries, including the USA, England, Denmark, and Germany, with interest in identifying effective strategies to improve the hospital-to-home transition $[3,4]$. A small but growing body of literature on digital health interventions (DHIs) demonstrates their promise in addressing this challenge by helping to modify cardiovascular risk factors and increasing adherence to guideline-directed therapy [5-9]. In particular, recent digital health studies have shown a trend towards a reduction in cardiovascular-related re-hospitalizations and emergency department visits after acute coronary syndrome, as well as a reduction in the all-cause 30 -day readmission rate of heart failure patients $[8,10,11]$.

A key unanswered question is whether DHI use in cardiovascular patients is influenced by sociodemographic factors [7]. Prior studies that have assessed sociodemographic predictors of DHI use have done so within the community or primary care setting [12-16]. In this patient population, 
sociodemographic factors such as age, sex, race, marital status, educational level, and income/employment status have been investigated, demonstrating conflicting findings and a concern that digital health may be least used by patients who need it most [12-17]. Little is known about whether DHIs that are targeted towards secondary prevention or hospital readmission reduction are similarly influenced by such factors [7, 18-20]. Given the recent flood of investment in cardiovascular digital health and the championing of digital health as a way to tackle gaps in secondary prevention of cardiovascular disease, it is critical that potential sociodemographic disparities in DHI use are explored and mitigated to avoid exacerbating already present health disparities [21]. Our study thus leverages smartphone app analytics data to identify sociodemographic predictors of DHI use in patients recovering from AMI using the Corrie Health Digital Platform ("Corrie").

Corrie is a DHI targeting self-management in type 1 AMI patients during the hospital-to-home transition [18]. In the Myocardial infarction, COmbined-device, Recovery Enhancement (MiCORE) Study, we found that AMI patients using Corrie had a significantly reduced rate of all-cause unplanned 30-day readmissions compared with a historical comparison group [22]. Here, we sought to characterize the daily use patterns of DHI features and examine associations with sociodemographic characteristics.

\section{Methods}

\section{Study Participants}

The design of the MiCORE study (NCT03760796) has been described previously [18]. Briefly, the study was a multi-center, prospective study with a historical comparison group investigating the effect of Corrie in reducing 30-day readmissions after AMI. From October 1, 2016 to April 14, 2019, 200 type 1 AMI patients ages 18 years and older who owned a smartphone, spoke English, and had no hemodynamic instability or severe impairment that would interfere with smartphone use were enrolled in the Corrie intervention group. Enrollment occurred at four hospitals including Johns Hopkins Hospital, Johns Hopkins Bayview Medical Center, Reading Hospital, and Massachusetts General Hospital. Patients were provided with the Corrie iPhone application and Apple Watch as early as possible during their hospitalization. After the pilot phase, patients additionally received a Bluetooth-enabled blood pressure monitor on enrollment. Notably, our study was not limited to iPhone users; to mitigate selection bias, patients who did not own a Corrie-compatible iPhone were provided a loaner iPhone, and all analyses were adjusted by this variable. Patients had access to the intervention while hospitalized and for 30 days post-hospital discharge. The protocol was reviewed and approved by the
Johns Hopkins University School of Medicine Institutional Review Board (IRB00099938) and other participating sites.

The Corrie platform was divided into four features: (1) medication management, (2) vital signs monitoring, (3) educational videos and articles, and (4) follow-up appointment scheduling assistance. Patients were encouraged to review educational content and schedule follow-up appointments while inpatient. App analytics data were extracted from the backend Corrie Data Platform, which sourced data through Apple HealthKit, ResearchKit, and CareKit. Among Corrie users, 34 patients were excluded because they participated in the MiCORE study before the ability to capture smartphone app analytics data was developed (Fig. 1), smartphone analytics data were missing due to the app never being put to use ( $n=11)$, account not being created $(n$ $=2)$, technical failure $(n=11)$, or no use of features of interest in the study period $(n=9)$, resulting in a total of 133 patients included in this analysis. There were no significant differences in age, sex, or race between patients who were included compared with those who were excluded (Supplementary Table 1).

\section{Assessment of Feature Use}

Our primary outcome was use of the vital sign monitoring and medication tracking features. We focused our primary outcome on these two features since the remaining two features (education and follow-up appointment scheduling) were heavily targeted towards use prior to discharge instead of post-discharge.

DHI use was defined as the total number of days that patients used any aspect of the medication or vital signs feature. Use of each feature was first individually categorized into tertiles from the data distribution. Since we found that use of the medication feature was highly correlated with use of the vital signs feature $(p<0.001)$, we further derived a composite measure of DHI use (Supplementary Table 2). Patients in the lowest tertile of use of both features were considered low DHI users, while those who were in the highest tertile of both features were considered high DHI users. All other patients were considered moderate DHI users. Additionally, a heat map was created to visually assess feature use by day for each patient over the 30-day period.

\section{Sociodemographic and Clinical Characteristics}

Demographic, clinical, and digital health characteristics were collected on each participant from the electronic medical record and patient self-report questionnaires. Exposures of interest included age, sex, race, marital status, and insurance status.

Potential predictors that were considered included income and education level; however, they did not trend towards correlation with DHI use in bivariate analysis and were thus not included in the multivariable adjusted model. 


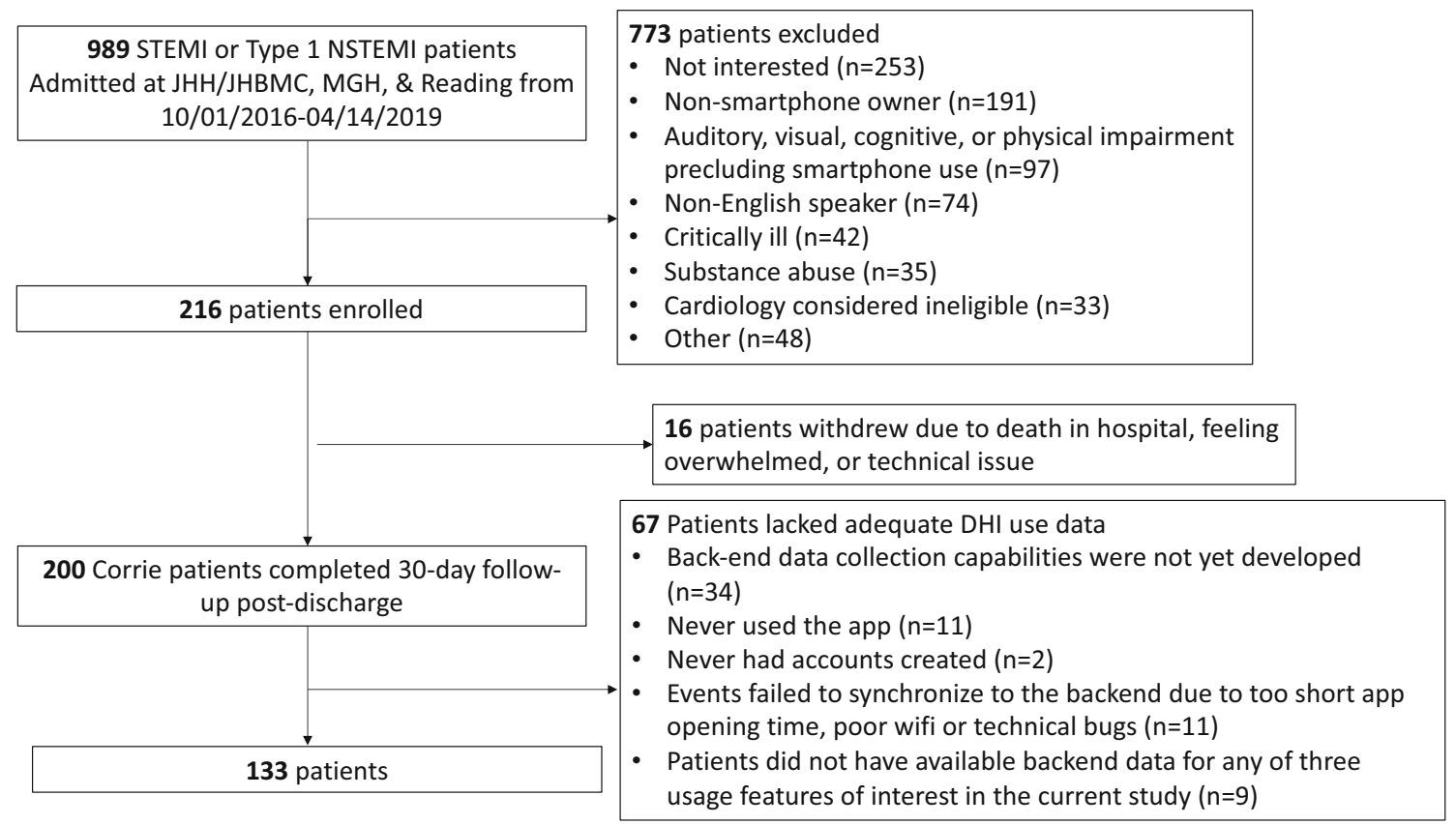

Fig. 1 Screening and enrollment of study participants

\section{Statistical Analysis}

Sociodemographic and digital health characteristics were compared among trichotomous DHI use categories using analysis of variance for continuous data and Chi-squared tests for categorical variables. We conducted ordered logistic regression models to examine the association between high DHI use and each sociodemographic factor including age, sex, race, marital status, and insurance status. All analyses were initially adjusted for age, sex, and race, followed by additional adjustment for loaner iPhone and treatment with CABG as a surrogate measure of disease severity (Table 4). Analyses that included use of the vital signs feature were additionally adjusted for presence of Bluetooth-enabled blood pressure monitor.

The proportional odds assumption was examined by using both the likelihood ratio test and the Brant test. Interactions between risk factors and other covariates were assessed in the fully adjusted models. Exploratory analyses were performed to assess for association between DHI use and hospital readmissions. All statistics were performed using the Stata version 15.1 software package (StataCorp LLC). A heat map was created using Displayr, Inc.

\section{Results}

Participant demographic and major clinical characteristics are shown in Table 1. The mean age was 58.3 years (SD 11.5, range 30 to 89 years), with $31 \%$ female, $68 \%$ White, and $62 \%$ married. Private health insurance was owned by 53\% of participants and 33\% of patients had Medicare. Patients who did not have private insurance or Medicare either had Medicaid or were uninsured.

Our study sample's mean calculated BMI was $30.9 \mathrm{~kg} /$ $\mathrm{m}^{2}$ (SD 5.9) and median length of inpatient admission was 5 days (IQR 3-11). Forty-two percent of patients had a diagnosis of ST-Elevation Myocardial Infarction (STEMI), and $29 \%$ of patients were treated with CABG. Patients had access to Corrie for a median of 2 days from enrollment to discharge (IQR 1-6) in addition to the 30day post-discharge period, and $40 \%$ of patients used a loaner iPhone to access the intervention. Over the study period, participants accessed the medication tracking feature a median of 11 unique days (IQR 2-27) and accessed the vital signs feature a median of 7 unique days (IQR 125) (Table 2).

Each patient's use of the medication and vital signs features by day post-discharge is shown in the heat map in Fig. 2. Close to a third (30\%) of patients used the medication feature for 22 or more days and also used the vital signs feature for 18 or more days (subsequently categorized as "high composite DHI use"; see Table 2). A third (33\%) of patients used the medication feature for less than 3 days and vital signs feature for less than 2 days ("low composite DHI use"). There was more use in the first half of the month compared with the second $(50 \%$ vs $40 \%$ of patient-days and $37 \%$ vs $29 \%$ of patient-days for the medication and vital signs features, respectively). Seventeen patients used the vital 
Table 1 Clinical and digital health characteristics of Corrie participants $(n=133)$

Characteristics during hospital admission

Clinical characteristics

\begin{tabular}{|c|c|}
\hline Age, years, mean $\pm \mathrm{SD}$ (range) & $58.3 \pm 11.5(30-89)$ \\
\hline Age $65+, \mathrm{n}(\%)$ & $43(32.3)$ \\
\hline Female, n (\%) & $41(30.8)$ \\
\hline White race, $\mathrm{n}(\%)$ & $91(68.4)$ \\
\hline \multicolumn{2}{|l|}{ Health insurance status } \\
\hline Private health insurance, $\mathrm{n}(\%)$ & $70(52.6)$ \\
\hline Medicare, n (\%) & $44(33.1)$ \\
\hline Medicaid/self-pay, n (\%) & $19(14.3)$ \\
\hline Annual income ${ }^{\mathrm{a}}$, median dollars (IQR) & $72,000(33,500-114,500)$ \\
\hline Years of education ${ }^{\mathrm{b}}$, mean $\pm \mathrm{SD}$ & $14.7 \pm 3.7$ \\
\hline Married $^{\mathrm{c}}, \mathrm{n}(\%)$ & $82(61.7)$ \\
\hline Body mass index, $\mathrm{kg} / \mathrm{m}^{2}$, mean $\pm \mathrm{SD}$ & $30.9 \pm 5.9$ \\
\hline Current/former smoking status, n (\%) & $69(51.9)$ \\
\hline Length of admission, median days (IQR) & $5(3-11)$ \\
\hline Diagnosis of STEMI, n (\%) & $56(42.1)$ \\
\hline Patients requiring $\mathrm{CABG}, \mathrm{n}(\%)$ & $38(28.6)$ \\
\hline \multicolumn{2}{|l|}{ igital Health characteristics } \\
\hline mHealth literacy score out of $40^{\mathrm{d}}$, mean $\pm \mathrm{SD}$ & $31.0 \pm 6.6$ \\
\hline Time with Corrie during admission, median days (IQR) & $2(1-6)$ \\
\hline Patients with loaner iPhone, n (\%) & $53(39.9)$ \\
\hline Patients given Bluetooth-enabled BP monitor, n (\%) & $108(81.2)$ \\
\hline
\end{tabular}

$S D$ standard deviation, $I Q R$ interquartile range, $S T E M I$ ST-elevation myocardial infarction, $C A B G$ coronary artery bypass grafting, $B P$ blood pressure

${ }^{\text {a }} n=80$ for income

${ }^{\mathrm{b}} n=116$ for years of education

${ }^{\mathrm{c}} n=128$ for marital status

${ }^{\mathrm{d}} n=111$ for mHealth literacy

signs feature almost every day (missed $0-1$ days) and 25 patients used the medication feature almost every day (missed 0-1 days). Four of these patients used both features daily.

Table 3 shows the sociodemographic characteristics of each group of patients, categorized by use of individual features and composite DHI use. Being married or having private insurance was significantly associated with high use of the vital signs feature, medication feature, and composite use of the DHI overall.

\section{Multivariable-Adjusted Associations with High Composite DHI Use}

After adjusting for age, sex, and race, married patients had higher odds (OR: 2.40, 95\% CI: 1.18-4.88) of being in the high composite DHI use group compared with those who were unmarried (see model 1; Table 4). Similarly, patients with private insurance had higher odds (OR: $3.57,95 \%$ CI: $1.22-10.41$ ) of being in the high composite DHI use group compared with those without. When additionally adjusted for presence of loaner iPhone and treatment with $\mathrm{CABG}$, the association persisted for married status (OR: 2.12, 95\% CI: 1.02-4.39). While the OR remained $>2$, the confidence interval no longer excluded 1.0 for private insurance (OR: $2.62,95 \%$ CI: $0.81-$ 8.46). In contrast, age, sex, and race were not significantly associated with DHI use before or after adjustment with covariates (Table 4).

Income and education level did not trend towards correlation with DHI use in bivariate analysis and were thus not included in the multivariable adjusted model.

\section{Exploratory Analyses}

Raw readmission data showed a general trend of decreasing readmissions as DHI use increased, with patients in the high composite DHI use group having the lowest rate of 30-day readmissions (Table 2). 
Table 2 DHI use and readmissions 30-day post-discharge

DHI use during 30-day post-discharge period

\begin{tabular}{ll}
\hline $\begin{array}{l}\text { Time spent on medication feature, median days (IQR) } \\
\text { Tertiles of medication feature use }\end{array}$ & $11(2-27)$ \\
1st tertile (<3 days), $\mathrm{n}(\%)$ & $47(35.3)$ \\
2nd tertile (3-22 days), n (\%) & $44(33.1)$ \\
3rd tertile ( $\geq 22$ days), $\mathrm{n}(\%)$ & $42(31.6)$ \\
Time spent on vital signs feature, median days (IQR) & $7(1-25)$ \\
Tertiles of vital signs feature use & \\
1st tertile (<2 days), $\mathrm{n}(\%)$ & $51(38.3)$ \\
2nd tertile (2-18 days), $\mathrm{n}(\%)$ & $38(28.6)$ \\
3rd tertile ( $\geq 18$ days), $\mathrm{n}(\%)$ & $44(33.1)$ \\
Number of patients in composite DHI use groups & $44(33.1)$ \\
Low composite use, $\mathrm{n}(\%)$ & $49(36.8)$ \\
Moderate composite use, $\mathrm{n}(\%)$ & $40(30.1)$ \\
High composite use, $\mathrm{n}(\%)$ & $12(9.0)$ \\
30-day all cause readmissions, $\mathrm{n}(\%)$ & $4(9.1)$ \\
Low composite DHI use, $\mathrm{n}(\%)$ & $6(12.2)$ \\
Moderate composite DHI use, $\mathrm{n}(\%)$ & $2(5.0)$ \\
High composite DHI use, $\mathrm{n}(\%)$ &
\end{tabular}

$D H I$ digital health intervention, $I Q R$ interquartile range

\section{Discussion}

\section{DHI Use Is Similar by Age, Sex, and Race in AMI Patients}

This study sought to identify if there are sociodemographic factors associated with the use of Corrie, a DHI developed to empower AMI patients in adhering to guideline-directed medical therapy and to reduce 30-day readmissions. Notably, we found that DHI use was similar by age, sex, and race, suggesting a potential role for DHIs to help achieve equity in cardiovascular health. We also found that some sociodemographic characteristics, marital and private insurance status, were associated with high DHI use after accounting for age, sex, and race. After additionally adjusting for loaner iPhone, Bluetooth-enabled blood pressure monitor, and $\mathrm{CABG}$ as a measure of disease severity, marital status remained significant while private insurance showed a trend, highlighting two areas that require further investigation.

The digital health literature is beginning to show that patient outcomes may improve with increased use of DHIs for secondary prevention. Widmer and colleagues demonstrated improved weight loss with DHI use in a dose-dependent relationship among patients following percutaneous coronary intervention, while a study conducted by Park and colleagues among heart failure patients found a trend towards less frequent use of a $\mathrm{DHI}$ in readmitted patients compared with non- readmitted patients $[23,24]$. Given the potential correlation between high DHI use and improved outcomes, our study extends prior digital health research by exploring sociodemographic characteristics that could predispose patients to higher or lower DHI use in a high risk, postdischarge cardiac patient population.

A key sociodemographic variable of interest has been age. There has been concern in the literature that seniors use digital health at low rates $[25,26]$. Some studies even suggest that the positive benefits of some digital health interventions, such as electronic symptom monitoring, may not extend to patients above the age of 70 [27]. Yet, other studies looking at primary prevention have found older patients to have the highest rates of DHI use [12, 14-16, 28]. Our findings suggest that DHIs are a tool that can be used for readmission reduction after AMI in patients of all ages. It should be noted that barriers to use related to age were considered during our initial DHI design. As the AMI population typically includes older adults, we collaborated with Apple Inc. to build a user-friendly interface that optimized color contrast, utilized larger buttons and text size, and minimized the number of screens to navigate through each feature. Our results suggest that with early consideration of patient population characteristics and iterative design, DHIs can be a suitable and highly utilized tool for elderly patients to independently and safely manage their own care. Our results also raise the question of whether patients behave differently when presented with a DHI for readmission reduction than when presented with a DHI for primary prevention, and if hospital discharge may be a particularly opportune time in a patient's care to start a patient on a DHI.

Second, sex and race are factors that are known to have outcome disparities in cardiovascular disease [29]. Our finding that DHI use is not significantly different across sex or race in the context of post-discharge self-management differs from prior evidence that looked at digital health use for general cardiac risk factor management $[12,28]$. This suggests that DHIs focused on readmission reduction may be a way to equitably improve adherence to guideline-directed medical therapy post-discharge for patients regardless of sex and race.

\section{The Presence of a Caregiver May Make Patients More Likely to Be High DHI Users}

Our study shows that the presence of a spouse, used here as a surrogate for a caregiver, is associated with patients having high DHI use. Although the confidence interval was relatively wide (1.02-4.39), our results suggest that caregivers may encourage patients to engage more frequently with a given DHI and ultimately adhere to their care plan. These results are in agreement with the current body of literature, which suggest that DHIs that involve caregivers improve cardiac medication adherence and may be effective in reducing re-hospitalizations [30-32]. Further investigation with a larger sample size and 
a

\begin{tabular}{c|l|}
\cline { 2 - 2 } $\begin{array}{c}\text { Vital Signs Feature Use Over } 30 \text { Days } \\
\text { Days Post-Discharge }\end{array}$ & $\begin{array}{l}\text { LEGEND } \\
\text { Feature used } \\
\text { Feature not used }\end{array}$ \\
\hline
\end{tabular}

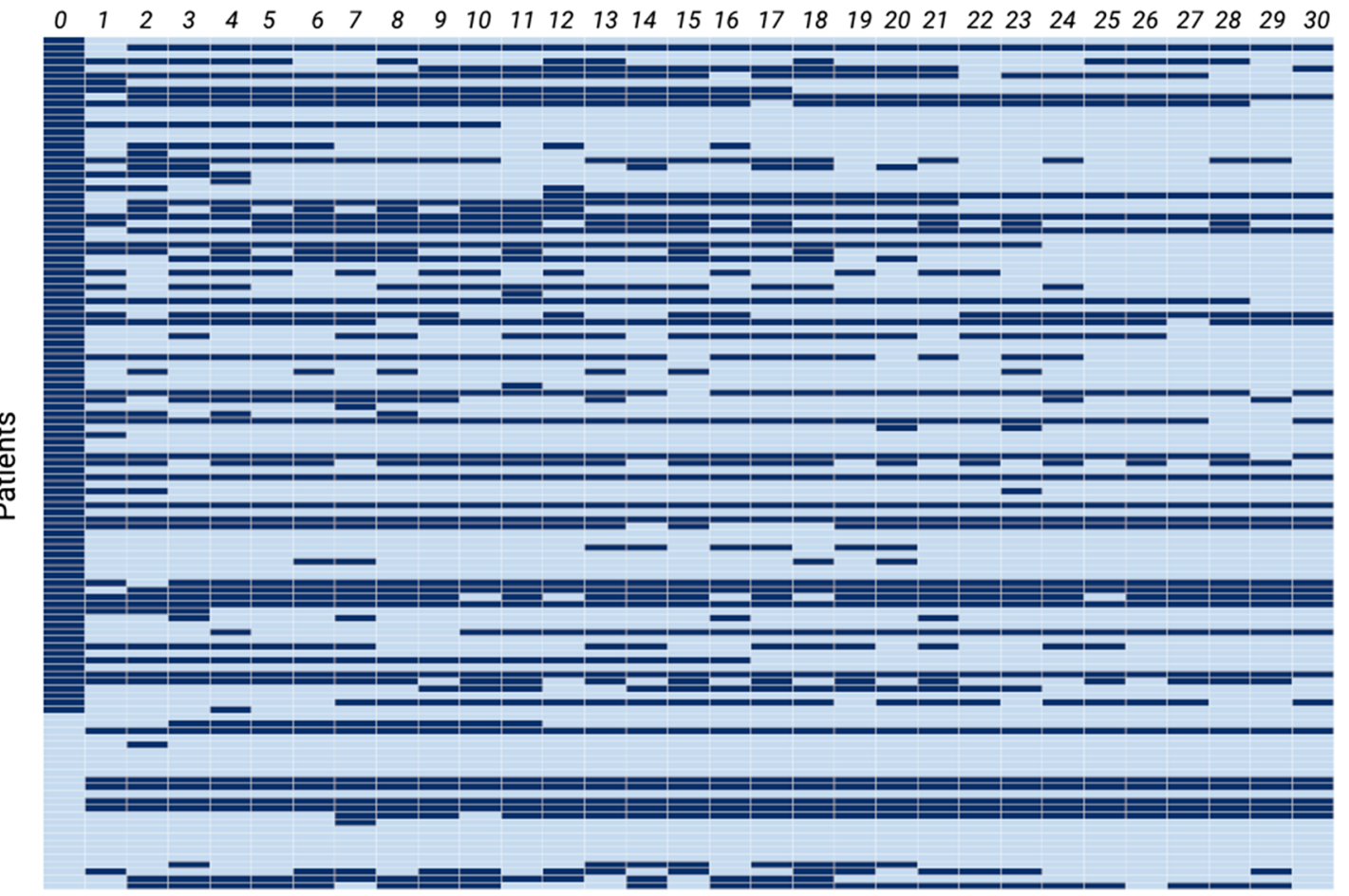

b

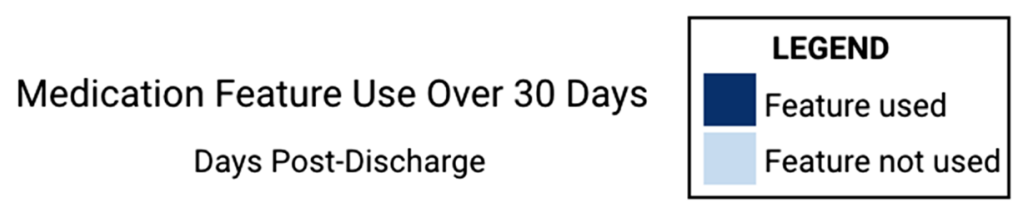

$\begin{array}{llllllllllllllllllllllllllllllll}0 & 1 & 2 & 3 & 4 & 5 & 6 & 7 & 8 & 9 & 10 & 11 & 12 & 13 & 14 & 15 & 16 & 17 & 18 & 19 & 20 & 21 & 22 & 23 & 24 & 25 & 26 & 27 & 28 & 29 & 30\end{array}$

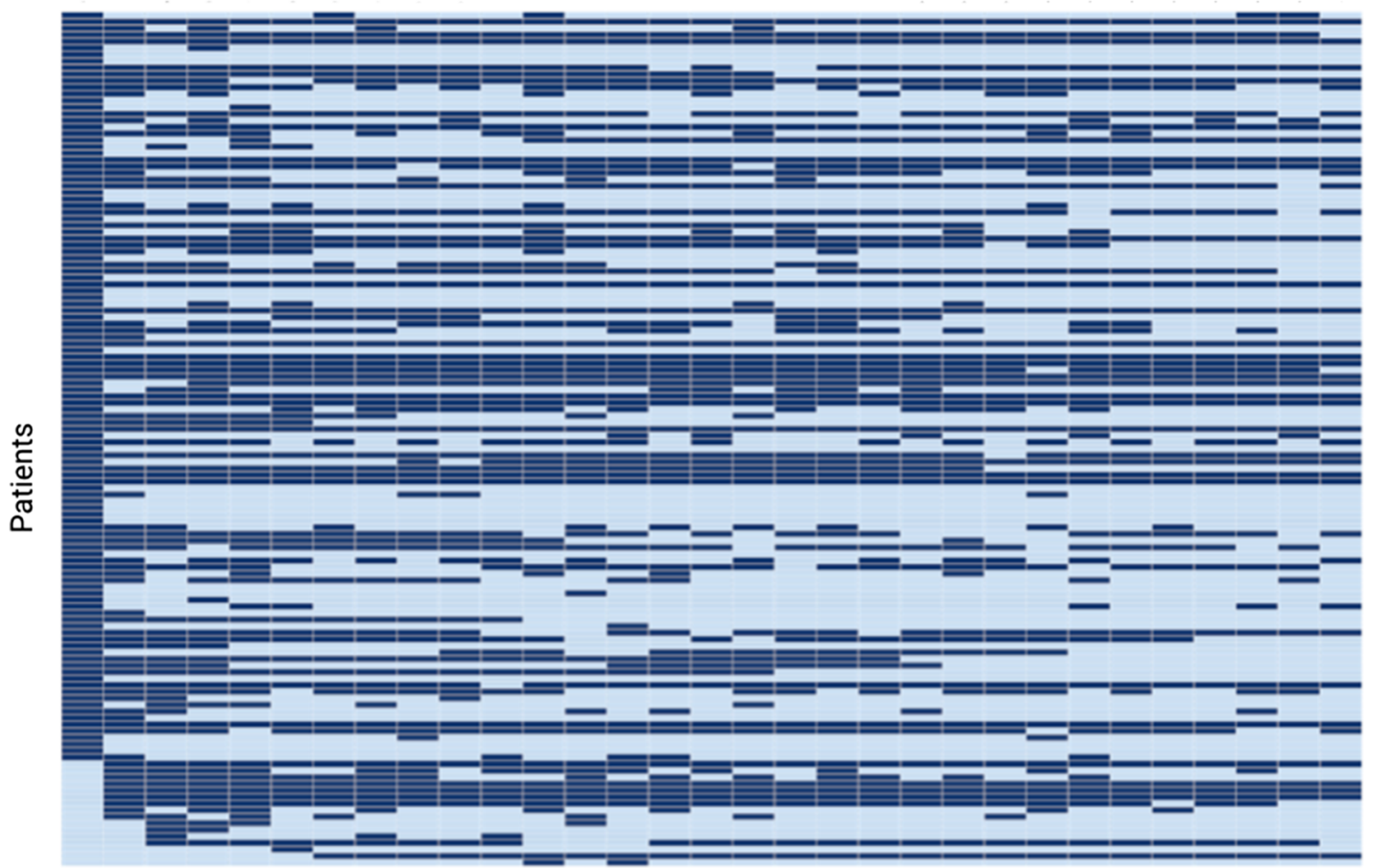

Fig. 2 Medication feature $(n=130)$ and vital signs feature $(n=121)$ use over 30 days post-discharge

Springer 
direct assessment of the presence/absence of a caregiver is needed to confirm these findings.

\section{The Influence of Socioeconomic Status on DHI Use Merits Further Investigation}

The correlation or lack thereof between insurance status, education level, and income with DHI use merits further investigation, particularly as these three markers may be surrogates for socioeconomic status. Previous studies have identified decreased DHI use in people of low socioeconomic status, defined by income stratification, in the setting of primary prevention $[6,25,33]$. These studies raise the specter of DHI implementation potentially worsening health disparities. However, in our analyses of patients post-discharge after myocardial infarction, neither income nor educational level was a predictor of DHI use. Unlike insurance status, they did not even trend towards correlation with DHI use. This leaves several possible interpretations. The first is that socioeconomic status may not influence use of a DHI to reduce readmissions, indicating that DHIs may have the potential to address health disparities by improving outcomes across all socioeconomic strata. Alternatively, a difference in DHI use by income, education level, or insurance may exist but we may not have been powered to detect it; in particular, we had less data available for education level $(n=116)$ and income $(n=82)$. Finally, our work may indicate that while insurance status may influence DHI use, it may be a poor proxy for socioeconomic status and there may be another, unexplored association between insurance and DHI use. Of note, the association between DHI use and insurance status became non-significant when it was additionally adjusted for presence of a loaner iPhone. Loaner iPhone may be a mediator of these two variables: i.e., Medicaid/uninsured patients may be more likely to require a loaner iPhone, and this may predispose them to decreased DHI use. In this context, while loaner iPhones may improve access to DHIs, there may be room to further optimize this solution to better meet the needs of all patients.

\section{One-Third of Patients Used the Medication Tracking and Vital Signs Features over $\mathbf{5 0 \%}$ of the Month}

Assessing DHI use by feature, as opposed to number of global interactions, can lead to insights related to participant retention. Our work suggests that features that support daily self-tracking of medication and vital signs measurements are well-tolerated and appreciated by many patients. However, as there was distinctly different use between the top third and bottom third of DHI users, it is clear that there may be opportunities to better support other patients. Importantly, since many sociodemographic factors were not significantly associated with DHI use, 
Table 4 Multivariable adjusted odds ratios (ORs) with 95\% confidence intervals of being in the highest DHI group versus the lower tertiles for various sociodemographic variables

\begin{tabular}{|c|c|c|c|c|c|c|}
\hline & \multicolumn{2}{|l|}{ Composite measure } & \multicolumn{2}{|l|}{ Vital signs feature } & \multicolumn{2}{|l|}{ Medication feature } \\
\hline & Model $1^{\mathrm{a}}$ & Model $2^{\mathrm{b}}$ & Model 1 & Model 2 & Model 1 & Model 2 \\
\hline Age & $1.00(0.97-1.02)$ & $0.98(0.95-1.01)$ & $1.00(0.96-1.02)$ & $0.98(0.95-1.01)$ & $1.00(0.97-1.03)$ & $0.99(0.96-1.02)$ \\
\hline Female sex & $0.51(0.25-1.01)$ & $0.60(0.29-1.25)$ & $0.61(0.30-1.21)$ & $0.70(0.34-1.45)$ & $0.52(0.26-1.03)$ & $0.59(0.29-1.23)$ \\
\hline White race & $1.30(0.66-2.55)$ & $1.22(0.60-2.48)$ & $1.33(0.68-2.64)$ & $1.34(0.65-2.77)$ & $1.23(0.64-2.50)$ & $1.10(0.55-2.21)$ \\
\hline Married status & $2.40(1.18-4.88)^{*}$ & $2.12(1.02-4.39)^{*}$ & $2.50(1.23-5.10)^{*}$ & $2.28(1.10-4.73)^{*}$ & $2.42(1.20-4.91)^{*}$ & $2.17(1.06-4.47)^{*}$ \\
\hline \multicolumn{7}{|l|}{ Insurance status } \\
\hline Medicaid/self-pay & 1.00 (Ref.) & 1.00 (Ref.) & 1.00 (Ref.) & 1.00 (Ref.) & 1.00 (Ref.) & 1.00 (Ref.) \\
\hline Private insurance & $3.57(1.22-10.41)^{*}$ & $2.62(0.81-8.46)$ & $3.80(1.25-11.57)^{*}$ & $3.41(1.01-11.52)^{*}$ & $3.89(1.30-11.64)^{*}$ & $2.78(0.85-9.05)$ \\
\hline Medicare & $1.53(0.42-5.55)$ & $1.15(0.29-4.50)$ & $1.46(0.38-5.64)$ & $1.22(0.29-5.17)$ & $1.83(0.49-6.77)$ & $1.49(0.38-5.84)$ \\
\hline
\end{tabular}

${ }^{\mathrm{a}}$ Model 1: adjusted for age, sex, and race, unless variable was included in model

${ }^{\mathrm{b}}$ Model 2: adjusted for age, sex, race, loaner iPhone, and treatment with CABG as a surrogate measure of disease severity. Analyses that included use of the vital signs feature were additionally adjusted for presence of Bluetooth-enabled BP monitor

*Reached formal significance

this raises the question if instead there are personality traits or attitudes that make some patients more likely to benefit from digital health than others. Future studies should consider holding focus groups with patients from high and low use groups to develop solutions tailored to unique digital health user personas. Finally, more frequent feature use in the first half of the month compared with the second is informative. One explanation is that there may be room for an intervention midway through the program to help patients stay on track. Alternatively, DHIs may function as "training wheels" for selfmanagement habits and may not be needed after a threshold amount of time. In order for DHIs to have the best chance of improving clinical outcomes, further research is needed to better identify and characterize trajectories of use over time.

\section{Strengths and Limitations}

To our knowledge, despite the well-established influence of sociodemographic factors on readmission risk [34, 35], our study is the first to explore the association of sociodemographic factors and use of a DHI to reduce hospital readmissions. However, we were limited by the number of patients in our study, particularly in examining the potential association between DHI use and readmission risk.

Our relatively small sample size and lack of power also required us to limit the number of covariates in our models. Age, sex, and race are well-established confounders that we were interested in a priori. In contrast, we knew little about insurance or marital status with respect to the confounding role they could play on the association between DHI use and age, sex, or race. For this reason, although our exposures of interest included age, sex, race, marital status, and type of insurance, we limited our covariates to age, sex, race, loaner iPhone, and CABG (as a measure of disease severity). Given the insights we have gained from the current analysis, it is reasonable for future studies with larger sample sizes to include marital and insurance status as covariates.

Another limitation is the skewness of the data. This resulted in tertiles of similar, but not equal, size being generated to describe feature use, which may have impacted our analyses. It also created groups with the potential for large intracategory variation, as the second tertile for both features was quite large (3-22 days for the medication feature and 2-18 days for the vital signs feature). This intra-category variation may have attenuated the statistical power in detecting an association and could also have contributed to the relatively higher readmission rate in the moderate composite DHI use group compared with the low composite DHI use group. We chose to categorize the data into tertiles in order to explore the association in a more clinically meaningful context, but future studies may consider modeling the use data by $\log$ transforming the data or performing non-linear modeling of continuous variables instead.

We also acknowledge potential for selection bias if patients included in the analysis were younger and healthier than those who were excluded. However, on analysis, we found no significant difference between the two groups in terms of all variables of interest including age, sex, race, insurance status, marital status, and disease severity. The use of Apple products also carries a risk of selection bias for iPhone users, which was mitigated by implementing the iPhone loaner program and adjusting all analyses by this variable. 
Finally, we chose to focus on the first 30 days post-discharge, due to 30-day readmission penalties from the Centers for Medicare and Medicaid Services [2]. However, longer follow-up is important and should be a focus of future work.

\section{Conclusion}

Ultimately, as smartphone ubiquity increases, DHIs can be a powerful tool to support evidence-based strategies to improve outcomes in cardiovascular patients. However, it is imperative to understand how DHIs may affect sociodemographic disparities, particularly at a time when COVID-19 has pushed healthcare systems towards rapidly adopting DHIs to fill emergent clinical needs [36].

First, elderly patients are at higher risk for COVID-19 infection and symptom severity; our work supports DHIs as a particularly timely intervention for patients of all ages to improve self-management skills and increase connection to care, while minimizing exposures [37]. Second, our work supports the use of DHIs to reduce hospital readmissions regardless of sex or race, strengthening the case for DHIs as a tool to help narrow outcome disparities in patients who experience AMI. Finally, future teams can further explore the association we found between caregiver presence and increased DHI use, as the caregiver-DHI combination may be an untapped, synergistic driver of adherence to guideline-directed therapy. In summary, our study is the proverbial "tip of the iceberg"; further research is needed in a larger study population to better understand the associations between sociodemographic factors, different patient attitudes and needs, DHI use, and outcomes of patients after AMI. Only then will we be able to create a strong foundation for DHIs to deliver on their promise of equitably improving cardiovascular health outcomes and optimizing care delivery during this pandemic and beyond.

Supplementary Information The online version contains supplementary material available at https://doi.org/10.1007/s12265-021-10098-9.

Acknowledgments We thank the MiCORE participants for their time and feedback, which was critical to DHI design and iterative improvements.

Author's Contributions LMS, FAM, and SSM contributed substantially to the conception and design of this work. LMS performed all analyses with significant support and guidance by JD. EMS, WEY, MAL, and RD were essential to data collection and the extraction protocol. LMS, JD, WEY, FAM, EMS, and SSM provided critical review of the manuscript. All gave final approval and agree to be accountable for all aspects of work ensuring integrity and accuracy. No human studies were carried out by the authors for this article.

Funding This study has received material support from Apple and iHealth and funding from the Maryland Innovation Initiative, Wallace H. Coulter Translational Research Partnership, Louis B.
Thalheimer Fund, and Johns Hopkins Individualized Health Initiative. Sponsors provided financial support and supplied materials, while the academic investigators had full rights in the design, collection, analysis, or interpretation of the data, and final approval of the manuscript for submission. EMS has received the following financial support for the research, authorship, and publication of this article: NIH/NHLBI T32 HL007024 Post-Doctoral Fellowship in Cardiovascular Epidemiology Institutional Training, NIH/NINR F31 NR017328, Ruth L. Kirschstein National Research Service Award and NIH/NINR T32 NR012704, PreDoctoral Fellowship in Interdisciplinary Cardiovascular Health Research. SSM has received research support from the American Heart Association (20SFRN35380046 and COVID19-811000), PCORI (ME-2019C1-15328), National Institutes of Health (P01 HL108800), Aetna Foundation, the David and June Trone Family Foundation, the Pollin Digital Innovation Fund, PJ Schafer Cardiovascular Research Fund, CASCADE FH, Apple, Google, and iHealth.

\section{Compliance with Ethical Standards}

Conflict of Interest Corrie Health, as described in this work, was developed by F.A.M., M.A.L., and S.S.M. FAM, MAL, and SSM are founders of and hold equity in Corrie Health, which intends to further develop the digital platform. This arrangement has been reviewed and approved by the Johns Hopkins University in accordance with its conflict of interest policies. SSM has served as a consultant to Akcea, Amgen, AstraZeneca, DalCor Pharmaceuticals, Esperion, Kaneka, Novo Nordisk, Quest Diagnostics, Regeneron, REGENXBIO, Sanofi, and 89bio. He is a co-inventor on a system to estimate LDL cholesterol levels, patent application pending. All other authors have no relevant relationships to disclose.

\section{References}

1. Piepoli, M. F., Corrà, U., Dendale, P., Frederix, I., Prescott, E., et al. (2016). Challenges in secondary prevention after acute myocardial infarction: A call for action. European Journal of Preventive Cardiology, 23(18), 1994-2006. https://doi.org/10.1177/ 2047487316663873.

2. Trends in hospital readmissions for four high-volume conditions, 2009-2013. Healthcare Cost and Utilization Project (HCUP), Agency for Healthcare Research and Quality. Retrieved November 16, 2019, from https://www.hcup-us.ahrq.gov/reports/ statbriefs/sb196-Readmissions-Trends-High-Volume-Conditions. jsp

3. Wiggins, B. S., Rodgers, J. E., \& DiDomenico, et al. (2013). Discharge counseling for patients with heart failure or myocardial infarction: A best practices model developed by members of the American College of Clinical Pharmacy's Cardiology Practice and Research Network based on the Hospital to Home (H2H) Initiative. Pharmacotherapy: The Journal of Human Pharmacology and Drug Therapy, 33(5), 558-580. https://doi.org/10.1002/phar.1231.

4. Wadhera, R. K., Maddox, K. E. J., Kazi, D. S., et al. (2019). Hospital revisits within 30 days after discharge for medical conditions targeted by the Hospital Readmissions Reduction Program in the United States: National retrospective analysis. The BMJ, 366. https://doi.org/10.1136/bmj.14563

5. Gandhi, S., Chen, S., Hong, L., Sun, K., \& Gong, et al. (2017). Effect of mobile health interventions on the secondary prevention of cardiovascular disease: Systematic review and meta-analysis. Canadian Journal of Cardiology, 33(2), 219-231. https://doi.org/ 10.1016/J.CJCA.2016.08.017. 
6. Senecal, C., Jay Widmer, R., Bailey, K., et al. (2018). Usage of a digital health workplace intervention based on socioeconomic environment and race: Retrospective secondary cross-sectional study. Journal of Medical Internet Research, 20(4). https://doi.org/10. 2196/jmir.8819.

7. Supervía, M., \& López-Jimenez, F. (2018). mHealth and cardiovascular diseases self-management: There is still a long way ahead of us. European Journal of Preventive Cardiology, 25(9), 974-975. https://doi.org/10.1177/2047487318766644.

8. Widmer, R. J., Allison, T. G., Lennon, R., Lopez-Jimenez, et al. (2017). Digital health intervention during cardiac rehabilitation: A randomized controlled trial. American Heart Journal, 188, 65-72. https://doi.org/10.1016/j.ahj.2017.02.016.

9. Kumar, S., Moseson, H., Uppal, J., \& Juusola, J. L. (2018). A diabetes mobile app with in-app coaching from a certified diabetes educator reduces A1C for individuals with type 2 diabetes. The Diabetes Educator, 44(3), 226-236. https://doi.org/10.1177/ 0145721718765650 .

10. Widmer, R. J., Allison, T. G., Lerman, L. O., \& Lerman, A. (2015). Digital health intervention as an adjunct to cardiac rehabilitation reduces cardiovascular risk factors and rehospitalizations. Journal of Cardiovascular Translational Research, 8(5), 283-292. https:// doi.org/10.1007/s12265-015-9629-1.

11. O'Connor, M., Asdornwised, U., Dempsey, M. L., Huffenberger, A., et al. (2016). Using telehealth to reduce all-cause 30-day hospital readmissions among heart failure patients receiving skilled home health services. Applied Clinical Informatics, 7(2), 238-247. https://doi.org/10.4338/ACI-2015-11-SOA-0157.

12. Serrano, K. J., Yu, M., Coa, K. I., Collins, L. M., \& Atienza, A. A. (2016). Mining health app data to find more and less successful weight loss subgroups. Journal of Medical Internet Research, 18(6). https://doi.org/10.2196/jmir.5473.

13. Goyal, S., Morita, P. P., Picton, P., Seto, E., Zbib, A., \& Cafazzo, J. A. (2016). Uptake of a consumer-focused mHealth application for the assessment and prevention of heart disease: The $<30$ days study. JMIR mHealth and uHealth, 4(1), e32. https://doi.org/10.2196/ mhealth.4730.

14. Edney, S., Ryan, J. C., Olds, T., Monroe, C., et al. (2019). User engagement and attrition in an app-based physical activity intervention: Secondary analysis of a randomized controlled trial. Journal of Medical Internet Research, 21(11), e14645. https://doi.org/10. 2196/14645

15. Mattila, E., Orsama, A. L., Ahtinen, A., Hopsu, L., Leino, T., \& Korhonen, I. (2013). Personal health technologies in employee health promotion: Usage activity, usefulness, and health-related outcomes in a 1-year randomized controlled trial. Journal of Medical Internet Research, 15(7). https://doi.org/10.2196/ mhealth.2557.

16. Garcia-Ortiz, L., Recio-Rodriguez, J. I., Agudo-Conde, C., et al. (2018). Long-term effectiveness of a smartphone app for improving healthy lifestyles in general population in primary care: Randomized controlled trial. JMIR mHealth and uHealth, 6(4). https://doi.org/10.2196/mhealth.9218.

17. Reiners, F., Sturm, J., Bouw, L. J. W., \& Wouters, E. J. M. (2019). Sociodemographic factors influencing the use of ehealth in people with chronic diseases. International Journal of Environmental Research and Public Health. MDPI AG. https://doi.org/10.3390/ ijerph 16040645

18. Spaulding, E. M., Marvel, F. A., Lee, M. A., \& Yang, et al. (2019). Corrie Health digital platform for self-management in secondary prevention after acute myocardial infarction. Circulation. Cardiovascular Quality and Outcomes, 12(5), e005509. https:// doi.org/10.1161/CIRCOUTCOMES.119.005509.

19. Auerbach, A. D. (2019, June 1). Evaluating digital health tools Prospective, experimental, and real world. JAMA Internal Medicine. https://doi.org/10.1001/jamainternmed.2018.7229.
20. Bonow, R. O., Grant, A. O., \& Jacobs, A. K. (2005). The cardiovascular state of the union: Confronting healthcare disparities. Circulation (Vol. 111, pp. 1205-1207). https://doi.org/10.1161/ 01.CIR.0000160705.97642.92

21. Ambrosetti, M., Abreu, A., Corrà, U., Davos, C. H., et al. (2020). Secondary prevention through comprehensive cardiovascular rehabilitation: From knowledge to implementation. 2020 update. A position paper from the Secondary Prevention and Rehabilitation Section of the European Association of Preventive Cardiology. European Journal of Preventive Cardiology, 14, 204748732091337. https://doi.org/10.1177/2047487320913379

22. Marvel FA, Spaulding EM, Lee M, et al. (2019). The Corrie myocardial infarction, combined-device, recovery enhancement (MiCORE) study: 30-day readmission rates and cost-effectiveness of a novel digital health intervention for acute myocardial infarction patients. QCOR 2019.

23. Park, C., Otobo, E., Ullman, J., Rogers, J., Fasihuddin, F., et al. (2019). Impact on readmission reduction among heart failure patients using digital health monitoring: Feasibility and adoptability study. JMIR Medical Informatics, 7(4), e13353. https://doi.org/10. 2196/13353

24. Widmer, R. J., Senecal, C., Allison, T. G., Lopez-Jimenez, F., et al. (2020). Dose-response effect of a digital health intervention during cardiac rehabilitation: Subanalysis of randomized controlled trial. Journal of Medical Internet Research, 22(2), e13055. https://doi. org/10.2196/13055.

25. Levine, D. M., Lipsitz, S. R., \& Linder, J. A. (2016, August 2). Trends in seniors' use of digital health technology in the United States, 2011-2014. JAMA. https://doi.org/10.1001/jama.2016.9124.

26. Mitchell, U. A., Chebli, P. G., Ruggiero, L., \& Muramatsu, N. (2018). The digital divide in health-related technology use: The significance of race/ethnicity. https://doi.org/10.1093/geront/ gny 138

27. Nipp, R. D., Horick, N. K., Deal, A. M., et al. (2020). Differential effects of an electronic symptom monitoring intervention based on the age of patients with advanced cancer. Annals of Oncology, 31(1), 123-130. https://doi.org/10.1016/j.annonc.2019.09.003.

28. Goyal, S., Morita, P. P., Picton, P., Seto, E., Zbib, A., \& Cafazzo, J. A. (2016). Uptake of a consumer-focused mHealth application for the assessment and prevention of heart disease. JMIR mHealth and uHealth, 4(1), e32. https://doi.org/10.2196/mhealth.4730.

29. Morris, A. A., Ko, Y., Hutcheson, S. H., \& Quyyumi, A. (2018). Race/ethnic and sex differences in the association of atherosclerotic cardiovascular disease risk and healthy lifestyle behaviors. Journal of the American Heart Association, 7(10). https://doi.org/10.1161/ JAHA.117.008250.

30. Wu, J. R., Mark, B., Knafl, G. J., Dunbar, S. B., Chang, P. P., \& DeWalt, D. A. (2019). A multi-component, family-focused and literacy-sensitive intervention to improve medication adherence in patients with heart failure-A randomized controlled trial. Heart \& Lung. https://doi.org/10.1016/j.hrtlng.2019.05.011.

31. Piette, J. D., Marinec, N., Janda, K., Morgan, E., et al. (2016). Structured caregiver feedback enhances engagement and impact of mobile health support: A randomized trial in a lower-middleincome country. Telemedicine and e-Health, 22(4), 261-268. $\mathrm{https} / / /$ doi.org/10.1089/tmj.2015.0099.

32. Padula, M. S., D’Ambrosio, G. G., Tocci, M., D'Amico, R., et al. (2019). Home care for heart failure: Can caregiver education prevent hospital admissions? A randomized trial in primary care. Journal of Cardiovascular Medicine, 20(1), 30-38. https://doi. org/10.2459/JCM.0000000000000722.

33. Veinot, T. C., Ancker, J. S., Cole-Lewis, H., Mynatt, E. D., et al. (2019). Leveling up. Medical Care, 57, S108-S114. https://doi.org/ 10.1097/MLR.0000000000001032.

34. Philbin, E. F., Dec, G. W., Jenkins, P. L., \& DiSalvo, T. G. (2001). Socioeconomic status as an independent risk factor for hospital 
readmission for heart failure. American Journal of Cardiology, 87(12), 1367-1371. https://doi.org/10.1016/S0002-9149(01) 01554-5.

35. Havranek, E. P., Mujahid, M. S., Barr, D. A., Blair, I. V., et al. (2015). Social determinants of risk and outcomes for cardiovascular disease: A scientific statement from the American Heart Association. Circulation. Lippincott Williams and Wilkins. https://doi.org/10.1161/CIR.0000000000000228
36. Keesara, S., Jonas, A., \& Schulman, K. (2020). Covid-19 and health care's digital revolution. The New England Journal of Medicine. https://doi.org/10.1056/nejmp2005835.

37. Coronavirus (COVID-19). American Heart Association. Retrieved May 10, 2020, from https://www.heart.org/en/coronavirus

Publisher's Note Springer Nature remains neutral with regard to jurisdictional claims in published maps and institutional affiliations. 